\title{
A Facile Synthetic Method of Silver Nanoparticles with a Continuous Size Range from sub-10 $\mathrm{nm}$ to $40 \mathrm{~nm}$
}

\author{
Longhai Piao, Kyung Hoon Lee, ${ }^{\dagger}$ Byoung Koun Min, ${ }^{\dagger}$ Woong Kim, ${ }^{\S}$ Young Rag Do, ${ }^{\dagger}$ and Sungho Yoon ${ }^{\dagger}, *$ \\ Department of Chemistry, Kongju National University, Chungnam 314-701, Korea \\ ${ }^{\dagger}$ Department of Bio \& Nano Chemistry, Kookmin University, Seoul 136-702, Korea. ${ }^{*}$ E-mail: yoona@kookmin.ac.kr \\ ${ }^{\ddagger}$ Department of Clean Energy Research Center, Korea Institute of Science and Technology, \\ 39-1 Hawolgok-dong, Seongbuk-gu, Seoul 136-791, Korea \\ ${ }^{\S}$ Department of Materials Science and Engineering, Korea University, Seoul 136-713, Korea \\ Received September 4, 2010, Accepted October 30, 2010
}

\begin{abstract}
Size-controlled Ag nanoparticles (NPs) were prepared from the decomposition of $\mathrm{Ag}(\mathrm{I})$ carboxylates using ethanolamine derivatives as a reducing agent without an additional stabilizing agent. The size of the Ag NPs with a narrow size distribution (sub-10 $\mathrm{nm}$ to $c a .40 \mathrm{~nm}$ ) was controlled precisely by varying the processing parameters, such as the type of reducing agent and the chain length of the carboxylate in the Ag(I) carboxylate. The optical properties, surface composition and crystallinity of the Ag NPs were characterized by ultraviolet-visible spectroscopy, gas chromatography-mass spectrometry, thermal gravimetric analysis, transmission electron microscopy and X-ray diffraction.
\end{abstract}

Key Words: Silver nanoparticle, Nanocrystalline materials, Decomposition

\section{Introduction}

Nanoscale materials have attracted considerable interest due to their potential applications in the field of electronics, catalysis and pharmaceuticals etc. ${ }^{1-3}$ The chemical and physical properties of nanomaterials are remarkably different from those of the bulk form and are strongly dependent on their size and shape. ${ }^{4-6}$ Therefore, the reproducible preparation of NPs with the desired size and distribution is of enormous importance and an essential requirement for nanotechnology. Considerable efforts have been made to develop controlled and reproducible synthetic methods of NPs. ${ }^{7-13}$

Recently, there has been increasing demand for more compact, lightweight and flexible electronic devices. Inkjet printing in printable electronics is one of the most promising techniques for fulfilling such needs due to its ability to produce fine and complex patterns on a variety of substrate surfaces. ${ }^{14}$ The practical applications of such technology to generate conducting patterns of copper, ${ }^{15}$ silver ${ }^{16}$ and gold ${ }^{17}$ have been reported. Ag has become the most widely used material for produce conducting lines in industry because of the instability of copper and high cost of gold. Ag ink containing Ag NPs is often used to generate a Ag pattern using inkjet printing. Considering the diverse processing conditions and product requirements, precise control of the rheological properties and sintering temperature of the inks are essential, which have been reported to be strongly affected by the diameter of the Ag NPs. ${ }^{18-19}$ In this context, there is a need for a reproducible size-controlled synthetic route for Ag NPs. A range of methods have been reported to control the size of Ag NPs by varying the reaction conditions and/or the properties or configurations of the precursor used. ${ }^{20-25}$

Nakamoto et al. reported the synthesis of Ag NPs from the decomposition of a specially designed coordinated compound. ${ }^{26-29}$ The Ag NPs were prepared by the reduction of Ag(I) carboxylates with a reducing agent, triethylamine, under mild conditions, e.g. $80{ }^{\circ} \mathrm{C}^{26}$ The size (from $2.7 \mathrm{~nm}$ to $4.4 \mathrm{~nm}$ ) of the Ag NPs was strongly affected by the alkyl chain length of the carboxylate, while the reducing agent and other reaction conditions had a negligible effect. ${ }^{28}$ Although it is a facile method for producing monodisperse Ag NPs, the size range is too narrow (almost all the high-quality NPs were in the sub-10 nm size regime). In their later report, large NPs could be prepared using short chain length or branched carboxylates. However, the synthesized NPs had a broad size distribution. ${ }^{27,28}$ Considering the demand for Ag NPs of various sizes with a narrow distribution in printable electronics, it is important to advance this synthetic method to the $10 \sim 50 \mathrm{~nm}$ region.

This paper reports the size-controlled synthesis of Ag NPs from the reduction of $\mathrm{Ag}(\mathrm{I})$ carboxylates using ethanolamine derivatives as the reductant and also solvent simultaneously. Ag NPs ranging in size from sub-10 $\mathrm{nm}$ to $40 \mathrm{~nm}$ were synthesized by varying the chain length of the carboxylate and the type of ethanolamine.

\section{Experimental}

$\mathrm{AgNO}_{3}(99.8 \%)$ and $\mathrm{NaOH}$ (extra pure) were obtained from SAMCHUN CHEMICAL (Korea). Monoethanolamine (mEA) (99\%), diethanolamine (dEA) (99\%), triethanolamine (tEA) $(98 \%)$, myristic acid $\left(\mathrm{C}_{14}\right)(99 \%)$, palmitic acid $\left(\mathrm{C}_{16}\right)(95 \%)$ and stearic acid $\left(\mathrm{C}_{18}\right)(95 \%)$ were purchased from Sigma-Aldrich. All chemicals were used as received. The ultraviolet (UV)visible (vis) spectra were taken using a UV-vis spectrometer (X-ma2000, Human Corp., Korea) in the range of $300-800 \mathrm{~nm}$. Transmission electron microscopy (TEM) was performed on a FEI Tecnai F20 microscope operated at $100 \mathrm{kV}$. The average diameter and distribution of the Ag NPs were obtained from at least 100 particles from the TEM images. In the case of nonspherical NPs, the diameter was calculated from the mean value of the longest and shortest diameter of the particles. The 
crystallinity of the Ag NPs was analyzed by powder X-ray diffraction (XRD, Shimadzu, XRD-6000). Thermal gravimetric analysis (TGA, TA instrument STA-1500) was performed under flowing nitrogen gas at a heating rate of $10{ }^{\circ} \mathrm{C} / \mathrm{min}$. The surface composition was determined by Temperature Programmed Desorption or Decomposition Mass-Spectrometry (TPD-MS). The carboxylate surfactant which was chemisorbed on the surface of Ag NPs was desorbed when the sample was heated at a programmed rate in an inert atmosphere, and the nature of the chemisorbed species was characterized with mass spectrometric (MS) analysis (the details in the Supporting Information).

Ag (I) Carboxylate. $\mathrm{Ag}(\mathrm{I})$ myristate $\left(\mathrm{AgC}_{14}\right), \mathrm{Ag}(\mathrm{I})$ palmitate $\left(\mathrm{AgC}_{16}\right)$ and $\mathrm{Ag}(\mathrm{I})$ stearate $\left(\mathrm{AgC}_{18}\right)$ were synthesized according to the methodology reported in the literature. ${ }^{29}$ Briefly, 1 equiv. of the corresponding carboxylic acid was dissolved in an aqueous $\mathrm{NaOH}$ solution at $80^{\circ} \mathrm{C} .1$ equiv. of $\mathrm{AgNO}_{3}(a q)(10 \mathrm{~mL}, 2.5 \mathrm{M})$ was added dropwise to the carboxylate solution with vigorous stirring. The white precipitates were filtered and washed twice with de-ionized water and dried under reduced pressure.

Ag NPs. Ag(I) carboxylate ( $0.8 \mathrm{~g})$ was introduced to the corresponding ethanolamine $(15 \mathrm{~g})$. The reaction was performed at $130{ }^{\circ} \mathrm{C}$ with vigorous stirring for $15 \mathrm{~min}$. The synthesized NPs were precipitated with ethanol and centrifuged at $3500 \mathrm{rpm}$ for 5 min. The precipitates were washed 3 times with ethanol and finally dried overnight under reduced pressure. Interestingly, the concentration of $\mathrm{Ag}(\mathrm{I})$ carboxylate used had no significant effects to the sizes of the Ag NPs resulted in.

\section{Results and Discussion}

The Ag NPs were prepared from the decomposition of $\mathrm{AgC}_{18}$, $\mathrm{AgC}_{16}$ and $\mathrm{AgC}_{14}$ using mEA, dEA and tEA as reducing agents (Scheme 1, Table 1).

A clear solution was generated upon mixing the $\mathrm{Ag}(\mathrm{I})$ carboxylate in mEA and dEA. However, the flocculent suspension was observed in the mixture of $\mathrm{Ag}(\mathrm{I})$ carboxylate and $\mathrm{tEA} . \mathrm{Ag}(\mathrm{I})$ carboxylate (e.g. $\operatorname{Ag}(\mathrm{I})$ stearate) has a polymeric structure, which results in poor solubility in most non-coordinating solvents at ambient temperature. The polymeric structure can break when coordinating chemicals are introduced, such as thiol, primary and secondary amines etc.. It is difficult for bulky tEA to coordinate to $\mathrm{Ag}(\mathrm{I})$ carboxylate due to steric hindrance.

Each reaction mixture was treated thermally at $130{ }^{\circ} \mathrm{C}$ without any other additional solvent or stabilizing agent, resulting in a gradual color change. In the case of the mEA mixture, the color was changed gradually from a light yellow to brown and finally to gray. For dEA, the color was finally changed to a deep blue.

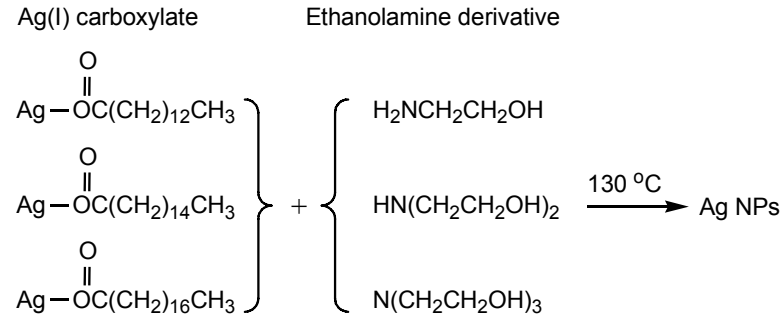

Scheme 1
Table 1. The reaction conditions and characterization of the Ag NPs

\begin{tabular}{|c|c|c|c|c|c|c|}
\hline Entry & Precursor & Reductant & $\begin{array}{l}\text { Yield } \\
(\%)^{a}\end{array}$ & $\begin{array}{c}\text { Amount of } \\
\text { surfactant } \\
(\%)^{b}\end{array}$ & $\begin{array}{l}\text { Domain } \\
\text { size } \\
(\mathrm{nm})^{c}\end{array}$ & $\begin{array}{l}\text { Diameter } \\
(\mathrm{nm})^{d}\end{array}$ \\
\hline 1 & $\mathrm{AgC}_{18}$ & $\mathrm{tEA}$ & 80 & 17.5 & 4.3 & $7.2 \pm 1.3$ \\
\hline 2 & $\mathrm{AgC}_{18}$ & dEA & 93 & 8.5 & 6.3 & $13.3 \pm 1.4$ \\
\hline 3 & $\mathrm{AgC}_{18}$ & $\mathrm{mEA}$ & 95 & 4.1 & 11.4 & $18.2 \pm 3.3$ \\
\hline 4 & $\mathrm{AgC}_{16}$ & tEA & 76 & 8.4 & 5.0 & $10.0 \pm 2.2$ \\
\hline 5 & $\mathrm{AgC}_{16}$ & dEA & 91 & 4.5 & 12.6 & $20.4 \pm 4.4$ \\
\hline 6 & $\mathrm{AgC}_{16}$ & mEA & 92 & 1.6 & 20.3 & $33.0 \pm 7.8$ \\
\hline 7 & $\mathrm{AgC}_{14}$ & tEA & 78 & 7.8 & 7.5 & $14.2 \pm 2.5$ \\
\hline 8 & $\mathrm{AgC}_{14}$ & dEA & 90 & 4.0 & 10.1 & $24.9 \pm 3.6$ \\
\hline 9 & $\mathrm{AgC}_{14}$ & mEA & 93 & 1.2 & 15.8 & $43.6 \pm 11.2$ \\
\hline
\end{tabular}

${ }^{a}$ The yields were calculated by comparing the metal content of $\mathrm{Ag}(\mathrm{I})$ carboxylate precursor and the Ag NPs. ${ }^{b}$ Measured with TGA. ${ }^{c}$ Calculated from Scherrer equation using the half-width of the (111) peaks of the Ag NPs. ${ }^{d}$ From TEM images.

When tEA was used, the deep brown color of the reaction mixture showed almost no change during the reaction. The change in the color of the reaction suggests a difference in the size of the Ag NPs produced. Each reaction was stopped when no additional color change was observed.

The UV-vis absorption spectra of samples $\mathbf{4 , 5}$ and $\mathbf{6}$ dispersed in an ethanol solution was measured to confirm the observed color change of the reaction mixture (Figure 1). ${ }^{31}$ The peak maxima of the adsorption bands shifted from $400 \mathrm{~nm}$ to $408 \mathrm{~nm}$, even though samples 4, 5 and 6 had been prepared from the $\mathrm{AgC}_{16}$ precursor but with a different reductant. From Mie theory, the increase in the size of the Ag NP induces a red shift of the Surface Plasmon Resonance (SPR) peaks, ${ }^{32}$ suggesting that the diameter of the Ag NPs decrease in the order of mEA, dEA and tEA.

To confirm the observation of the UV-vis spectra, TEM analysis of the Ag NPs and their size distribution histograms was performed (Figure 2). Samples 1, 2 and $\mathbf{3}$ were prepared from $\mathrm{AgC} 18$ with mEA, dEA and tEA as the reductant, respectively. The diameters of the NPs from samples $\mathbf{1}, \mathbf{2}$ and $\mathbf{3}$ were 18.2 (3.3) $\mathrm{nm}, 13.3(1.4) \mathrm{nm}$ and $7.2(1.3) \mathrm{nm}$, respectively, suggesting

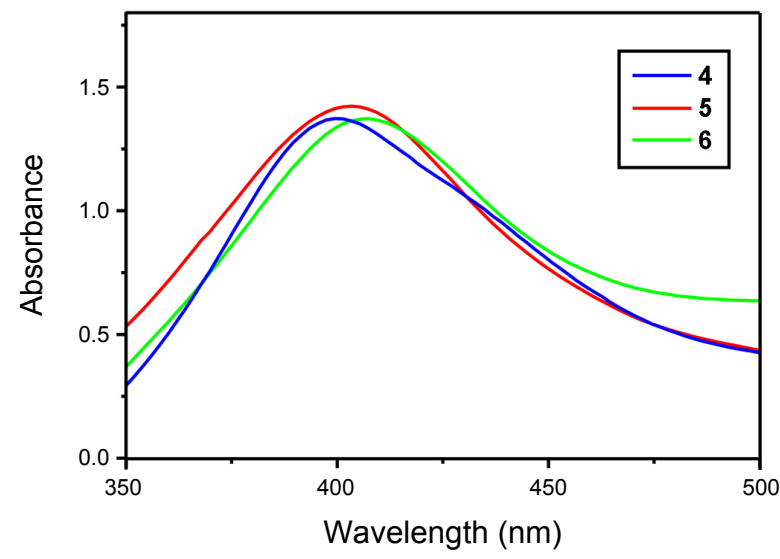

Figure 1. UV-vis absorption spectra of 4, 5 and $\mathbf{6}$ dispersed in the ethanol solution of PVP. 

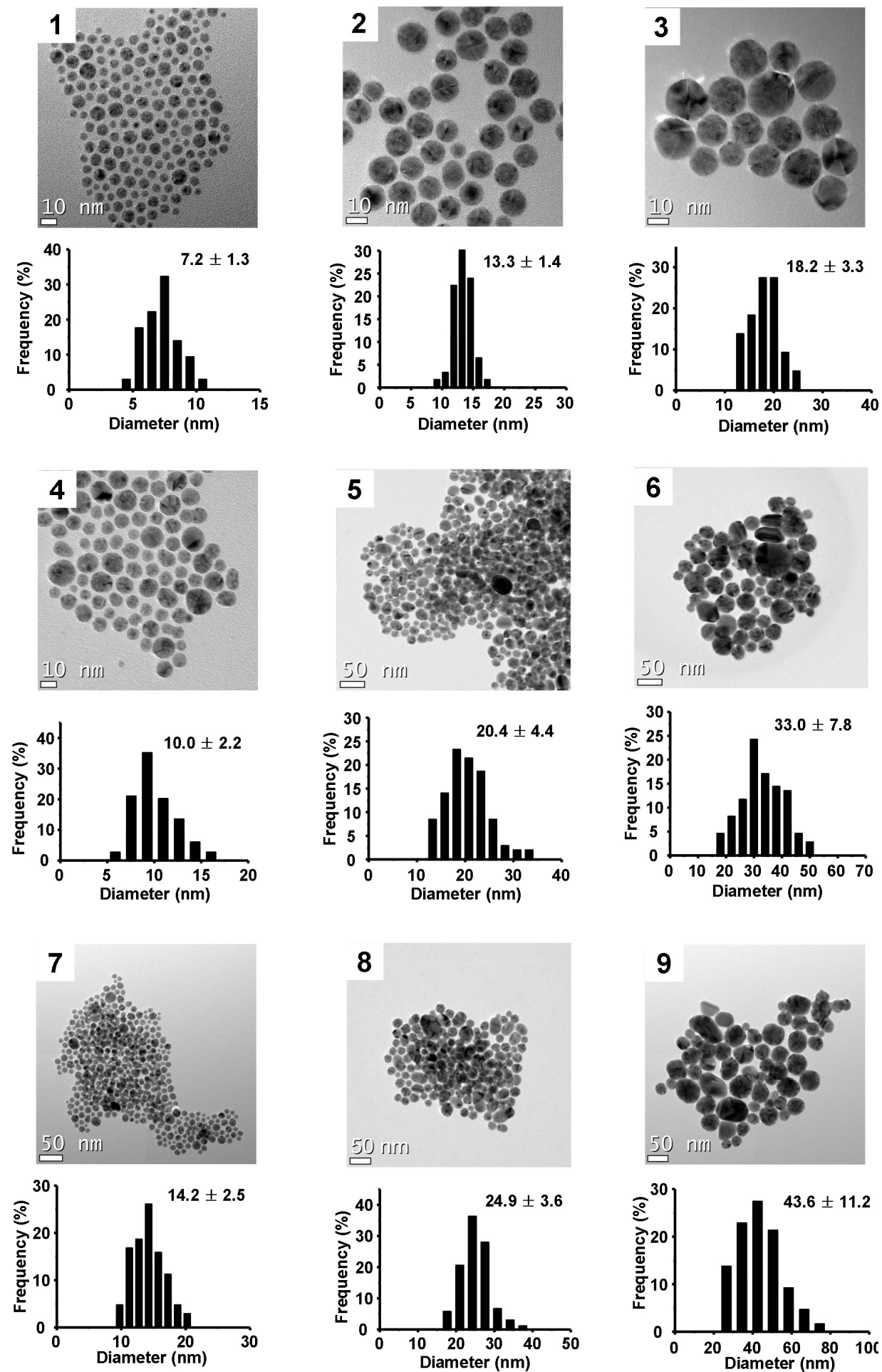

Figure 2. TEM images and the size distribution histograms of the Ag nanopartilces.

that the sizes of the Ag NPs were strongly influenced by the types of reducing agents. The size of the Ag NPs decreased in the order of mEAm, dEAm and tEAm as a reducing agent. Interestingly, the reducing agents had an apparent effect on the size of Ag NPs because Nakamoto et al. reported that the types of amines had negligible effect. ${ }^{28}$ They attempted several types of tertiary alkyamines and primary amines. None affected the size and size distribution of the NPs except for $n$-octylamine.
They attributed it to the weak coordination of the amines to the Ag nanoparticles during the growth process. Samples 1, 4 and 7 were prepared from $\mathrm{AgC}_{18}, \mathrm{AgC}_{16}$ and $\mathrm{AgC}_{14}$ with mEA as the reductant. The diameters of the NPs from samples 1, $\mathbf{4}$ and 7 were 7.2 (1.3) nm, 10.0 (2.2) nm and 14.2 (2.5) nm, respectively, suggesting that the sizes of the Ag NPs were also affected by the chain length of the carboxylate ligand of the $\mathrm{Ag}(\mathrm{I})$ carboxylate precursor; the size of the Ag NPs decreased with in- 


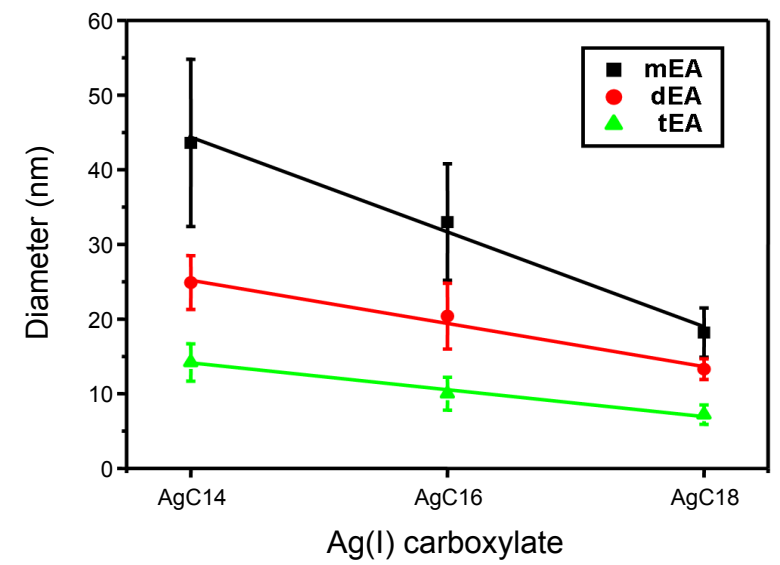

Figure 3. The relationship between the reductant, the chain length of the carboxylate ligand and the size of Ag NPs.

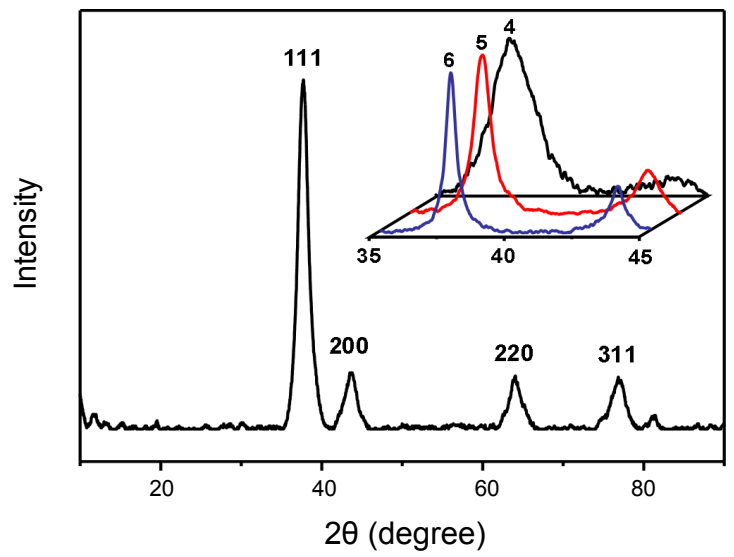

Figure 4. The XRD patterns of 6 . Inset shows the enlargements of the XRD patterns of 4 (black), 5 (red) and 6 (blue).

creasing alkyl chain length. Figure 3 shows the relationship between the reductant, chain length of the carboxylate ligand and the size of the Ag NPs. The size of the synthesized Ag NPs could be controlled from $7.2 \mathrm{~nm}$ to $43.6 \mathrm{~nm}$ by varying the chain length of the carboxylate ligand accompanied by a change in the reducing agent.

The crystallinity of the Ag NPs was examined by powder XRD analysis. The XRD patterns of sample 6 shows the characteristic (111), (200), (220) and (311) planes of face-centered cubic silver (Figure 4$){ }^{33}$ The average domain sizes were calculated from the half-width of the (111) reflection using the Scherrer equation (eq. 1) and are listed in Table 1. It should be noted that the line width of the (111) peaks of sample 4 was much broader than that of samples 5 and $\mathbf{6}$ (inset in Figure 5).

$$
\text { domain size }=\lambda /(\beta \cos \theta)
$$

where $\lambda$ is the wavelength of the X-rays (1.54 $\AA$ for $\mathrm{Cu} \mathrm{K} \alpha$ radiation), $\beta$ is the half-width of the (111) peak in radians and $\theta$ is the peak position.

The average domain sizes were smaller than the sizes of the Ag NPs observed by TEM, suggesting that the prepared Ag

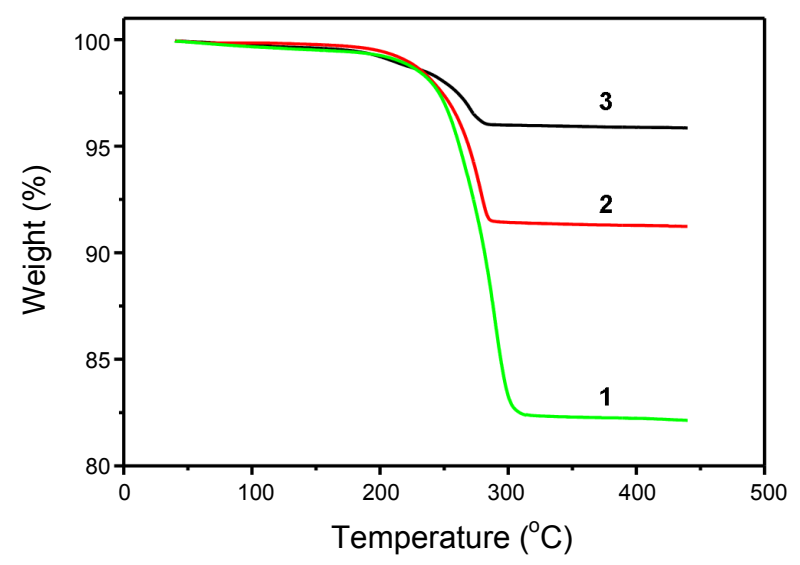

Figure 5. TGA curves of $\mathbf{1}$ (green), $\mathbf{2}$ (red) and $\mathbf{3}$ (black).

NPs were polycrystalline. The multi-twinned morphologies of the TEM images in Figure S1 also support this conclusion. The mean domain size increased with increasing NP size. Disordered multiply twinned structures are often observed in most noble-metal NPs. ${ }^{34-36}$ The physical and chemical properties of multi-crystalline NPs are different from their single-crystalline counterparts because of the different atomic energy sites on the crystallographic facets. Recently, it was reported that multi-crystalline Ag NPs with grain sizes down to $1 \mathrm{~nm}$ or less showed extremely bright and strongly enhanced Raman scattering compared to single crystalline Ag NPs. ${ }^{37}$

It was reported that the alkyl carboxylate derived from the $\mathrm{Ag}(\mathrm{I})$ carboxylate precursor acts as capping agent when the $\mathrm{Ag}(\mathrm{I})$ carboxylate decomposed with a tertiary amine or primary amine. Because there was no additional stabilizing agent, the ethanolamine would compete with the long chain carboxylate. Amines are believed to be weakly coordinating chemicals to Ag or Au NPs, even though the preparation of Ag NPs capped with amino-alcohols has been reported. ${ }^{38}$ The surface composition of the Ag NP was analyzed by TPD-MS and TGA. Only the corresponding carboxylate was detected by TPD-MS. No trace of the amines was observed, meaning that the Ag NP surface was capped only with the corresponding carboxylate. Table 1 lists the amounts of carboxylate attached to the surfaces of the Ag NPs, which were measured by TGA. (Figure 5) Below $350^{\circ} \mathrm{C}$, the organic component was removed completely, leaving only metallic Ag. The organic content decreased with increasing size of the Ag NPs (Figure S2). The yields were calculated by comparing the metal content of the $\mathrm{Ag}(\mathrm{I})$ carboxylate precursor with the Ag NPs (Table 1). The low yields of small NPs might be due to the loss of Ag NPs during the purification procedure.

\section{Conclusion}

The diverse Ag NPs with a spectral size window were synthesized from the decomposition of $\mathrm{Ag}(\mathrm{I})$ carboxylates using the derivatives of ethanolamine as a reducing agent without an additional stabilizing agent or solvent. The size of the Ag NPs was affected both by the type of reducing agent and the chain length of the carboxylate in the precursor. The size of the NPs 
decreased in the order of mEA, dEA and tEA as the reductant and increased with decreasing chain length of the carboxylate ligand. The Ag NPs synthesized ranged in size from sub-10 nm to $50 \mathrm{~nm}$. The surface of the Ag NPs was only capped by the corresponding carboxylate. Currently, our research is focused on determining methods for controlling the crystallinity from single to polycrystalline, and preparing Ag ink or paste from the Ag NPs described in this paper for applications to printable electronics.

Supporting Information Available. Figure S1, illustrating TEM images of the Ag NPs and Figure S2, showing the relationship between the reductant, the chain length of the carboxylate and the surfactant content of the Ag NPs.

Acknowledgments. This work was supported by the Korea Science and Engineering Foundation (KOSEF) grant funded by the Korea government (MEST) (No. R11-2005-048-00000-0) and a grant from the Ministry of Knowledge Economy, Republic of Korea. This work was supported in part by the faculty research program 2010 of Kookmin University in Korea and the research grant of Kongju National University in 2009.

\section{References}

1. Bell, A. T. Science 2003, 299, 1688.

2. Goddard, W. A.; Brenner, D. W.; Lyshevski, S. E.; Iafrate, G. J. Handbook of Nanoscience, Engineering, and Technology, 2nd ed.; CRC Press: FL, 2007.

3. Rosi, N. L.; Mirkin, C. A. Chem. Rev. 2005, 105, 1547.

4. Puntes, V. F.; Krishnan, K. M.; Alivisatos, A. P. Science 2001, 291, 2115.

5. Burda, C.; Chen, X. B.; Narayanan, R.; El-Sayed, M. A. Chem. Rev. 2005, 105, 1025 .

6. Milliron, D. J.; Hughes, S. M.; Cui, Y.; Manna, L.; Li, J. B.; Wang, L. W.; Alivisatos, A. P. Nature 2004, 430, 190.

7. Cozzoli, P. D.; Comparelli, R.; Fanizza, E.; Curri, M. L.; Agostiano, A.; Laub, D. J. Am. Chem. Soc. 2004, 126, 3868.

8. Zhang, W.; Qiao, X. J. Chen, Mater. Sci. Eng. B 2007, 142, 1.

9. Bronstein, L. M.; Kostylev, M.; Shtykova, E.; Vlahu, T.; Huang, X.; Stein, B. D.; Bykov, A.; Remmes, N. B.; Baxter, D. V.; Svergun, D. I. Langmuir 2008, 24, 12618 .

10. Scott, R. W. J.; Wilson, O. M.; Crooks, R. M. J. Phys. Chem. B 2005, 109, 692.

11. Briñas, R. P.; Hu, M. H.; Qian, L. P.; Lymar, E. S.; Hainfeld, J. F. J. Am. Chem. Soc. 2008, 130, 975.
12. Jana, N. R.; Gearheart, L.; Murphy, C. J. Langmuir 2001, 17, 6782.

13. Liu, Y. C.; Lin, L. H.; Chiu, W. H. J. Phys. Chem. B 2004, 108, 19237.

14. van Osch, T. H. J.; Perelaer, J.; de Laat, A. W. M.; Schubert, U. S. Adv. Mater. 2008, 20, 343

15. Rozenberg, G. G.; Bresler, E.; Speakman, S. P.; Jeynes, C.; Steinke, J. H. G. Appl. Phys. Lett. 2002, 81, 5249.

16. Smith, P. J.; Shin, D.-Y.; Stringer, J. E.; Derby, B.; Reis, N. J. Mater. Sci. 2006, 41, 4153 .

17. Chung, J.; Ko, S.; Bieri, N. R.; Grigoropoulos, C. P.; Poulikakos, D. Appl. Phys. Lett. 2004, 84, 801 .

18. Buffat, Ph.; Borel, J. P. Phys. Rev. A 1976, 13, 2287.

19. Luo, W.; Hu, W.; Xiao, S. J. Phys. Chem. C 2008, 112, 2359.

20. Komarneni, S.; Li, D. S.; Newalkar, B.; Katsuki, H.; Bhalla, A. S. Langmuir 2002, 18, 5959.

21. Skrabalak, S. E.; Wiley, B. J.; Kim, M.; Formo, E. V.; Xia, Y. N. Nano. Lett. 2008, 8, 2077.

22. Socol, Y.; Abramson, O.; Gedanken, A.; Meshorer, Y.; Berenstein, L.; Zaban, A. Langmuir 2002, 18, 4736.

23. Shankar, S. S.; Rai, A.; Ahmad, A.; Sastry, M. J. Colloid Interface Sci. 2004, 275, 496.

24. Kumar, A.; Vemula, P. K.; Ajayan, P. M.; John, G. Nat. Mater. 2008, 7, 236.

25. Hornebecq, V.; Antonietti, M.; Cardinal, T.; Treguer-Delapierre, M. Chem. Mater. 2003, 15, 1993.

26. Yamamoto, M.; Nakamoto, M. J. Mater. Chem. 2003, 13, 2064.

27. Kashiwagi, Y.; Yamamoto, M.; Nakamoto, M. J. Colloid Interface Sci. 2006, 300, 169.

28. Yamamoto, M.; Kashiwagi, Y.; Nakamoto, M. Langmuir 2006, 22, 8581.

29. Abe, K.; Hanada, T.; Yoshida, Y.; Tanigaki, N.; Takiguchi, H.; Nagasawa, H.; Nakamoto, M.; Yamaguchi, T.; Yase, K. Thin Solid Films 1998, 327-329, 524.

30. Cowdery-Corvan, P. J.; Whitcomb, D. R. In Photothermographic and Thermographic Imaging Materials, Handbook of Imaging Materials, 2nd ed.; Diamond, A. S., Weiss, D. S., Eds.; MarcelDekker: NY, 2002.

31. The NP was dispersed in an ethanol solution of polyvinylpyrrolidone.

32. Mishchenko, M. I.; Travis, L. D.; Lacis, A. A. Scattering, Absorption, and Emission of Light by Small Particles; Cambridge University Press: Cambridge, UK, 2002.

33. Smakula, A.; Kalnajs, J. Phys. Rev. 1955, 99, 1737.

34. Wang, Z. L. J. Phys. Chem. B 2000, 104, 1153.

35. Marks, L. D. Rep. Prog. Phys. 1994, 57, 603.

36. Tang, Y.; Ouyang, M. Nat. Mater. 2007, 6, 754.

37. Zheng, J.; Ding, Y.; Tian, B.; Wang, Z. L.; Zhuang, X. J. Am. Chem. Soc. 2008, 130, 10472.

38. Porta, F.; Krpetić, Ž.; Prati, L.; Gaiassi, A.; Scarì, G. Langmuir 2008, 24, 7061. 